\title{
On the Objective of Firms under Uncertainty with Stock Markets
}

\author{
Jean-Marc BONNISSEAU and Oussama LACHIRI*
}

January 30, 2004

\begin{abstract}
In a multi-period, multi-commodity economy with stock markets, we try to extend the work of Drèze (1974) to define the behavior of the firms. We exhibit first order necessary conditions for a constrained Pareto optimal allocation. The financial constraints lead to non-colinear supporting spot prices for the consumers at each node. Nevertheless, the firms are satisfying a first order necessary condition for profit maximization with respect to a price computed as the Drèze's prices. These prices are also consistent in the sense that the present values of the firms computed with the personal prices of the stockholders and with the Drèze's prices coincide when short sales are allowed. We also show that these conditions are simpler if we consider an allocation at which each consumer maximizes his preferences, when they are smooth. This allows us to give a formal definition for the objective of the firms, which extend the Drèze's criterion. We also discuss different definitions of constrained feasibility, and, we provide the related necessary conditions, which do not differ for the production sector.
\end{abstract}

JEL Classification Number : C60, D51, D52, D60

Key-words: Constrained Pareto optimality, multi-period, firm's behavior, incomplete markets, Drèze's criterion, stock market

*CERMSEM, UMR 8095, Université Paris 1 Panthéon-Sorbonne, Maison des Science économiques, 106 - 112 Boulevard de l'Hôpital, 75647 Paris Cedex 13, France, E-mail : Jean-Marc.Bonnisseau@univ-paris1.fr, lachiri@univ-paris1.fr 


\section{Introduction}

We consider an economy with time, uncertainty, several periods, several commodities at each state of nature and production. The transfer of wealth among periods and states of nature is possible through financial markets on the stocks of the firms. In this setting, it is known that the stockholders may disagree on the choice of the optimal production plans. Indeed, the share in the profit of a firm may have two different roles: an increase of the wealth in the future and a decrease on the risk among the states of nature. Since the shareholders may have different state prices, they may have a different evaluation of the production plans, and, thus they may have different optimal production plans.

A similar phenomenon appears when the firms have a strategic behavior on the market that is under imperfect competition. Indeed a conflict may appear for the agents between their interest as consumers of the products of the firms and as shareholders. Recent papers address the question of the objective of the firms in this setting (See Bejan (2003), Dierker, Dierker and Grodal (2001)). An important difference comes from the fact that the shareholders have fixed shares since there is no stock market in these papers whereas it is a mean to transfer wealth among states of nature in our model.

When market are complete or when the spanning condition holds true, then, even if the state prices are different at equilibrium, the no-arbitrage condition on the prices of the stocks implies that the optimal production plan is independent of the state prices. This is the case under the assumptions of Diamond's article (1967). Nevertheless, the spanning condition is restrictive since it means that the production sets are included in the linear space spanned by the equilibrium production plans. In other words, no firm has the possibility to implement a production plan, which is a true innovation in the sense that it is not a linear combination of existing production plans.

Our aim is to provide an objective for the firms in the general case without spanning condition. We follow the methodology initiated by Drèze (1974) and the program in the concluding remarks, which propose to extend the results to a multi-period, multi-commodity economy. Since we consider large firms, which have access to the stock markets, we do not impose any convexity assumption on the production sector. Indeed, the need for a large capital and the access to the stock market is justified by large fixed costs or increasing returns in the production.

The methodology is the following. Since it is hopeless to get a first best Pareto optimal equilibrium allocation, we consider a constrained Pareto optimal allocation. This means that the planner can redistribute the income among the consumers only through the financial market structure. Then, we exhibit the first order necessary conditions, and, we deduce from them a criterion for the firm's behavior. Note that this methodology is similar to the one of Guesnerie (1975) to define formally the marginal cost-pricing rule in a general equilibrium framework for non-convex firms.

In Bonnisseau-Lachiri (2002), we have considered an economy with several periods but only one perishable commodity at each state of nature. We show that the Drèze's criterion has a natural extension. This means that the firms are asked to satisfy a first order necessary condition of profit maximization with respect to the Drèze's prices. At each node, these prices are computed as a convex combination of the states prices of the stockholders, the coefficients being the shares held in the firm at this node. We cannot hope to have more than a necessary condition since the production sets are not supposed to be convex.

Furthermore, the non-convexity of the constrained attainable set excludes that the necessary conditions be enough to obtained a constrained Pareto optimal equilibrium allocation even if the productions sets are convex.

We refer to our previous paper for a discussion on the link with the criterion given in GrossmanHart (1979), which differs from the Drèze's one by the fact that the prices are computed with the fixed initial shares. In brief, this criterion satisfies a short-term weak optimality condition but it does not take into account the inter-temporal effect of the decisions.

In our general framework, the problem is not well posed. The notion of constrained feasible allocations is not well defined contrary to the case of a two period-one commodity economy. Indeed, commodity and stock prices enter into the definition and there are several possibilities to write formally 
that the transfers take place through the financial structure. Actually, the differences come from the possibility or not for the planner to change the spot or the stock prices.

Thus, we consider the largest possible constrained attainable set, which corresponds to the smallest constrained Pareto optimal set. This means that the planner has the possibility to choose the prices for the future periods. Nevertheless, we also investigate several variants of the definition of constrained feasibility. In each case, we give the necessary conditions at a constrained Pareto optimal allocation. The proofs are similar but simpler with smaller constrained attainable sets since we have less variables.

Note that the definition of constrained attainability is independent of the preferences of the agents like in Drèze (1974) or Magill-Quinzii (1996) but it is not directly comparable with the one in Geanakoplos et al. (1990), since in this last paper, the consumers are supposed to maximize the preferences under the prices set by the planner.

Contrary to our intuition, we cannot simply extend our previous results since the financial feasibility constraints with several commodities induce a deep change in the necessary conditions. Indeed, they give the existence of a supporting price for each consumer at the optimal allocation, but, if we consider the restrictions of these supporting prices at a given node, they are not always colinear. They only remain in a two dimensional space. This phenomenon disappears with only one commodity since the space of spot prices is of dimension 1. This fact remains true whatever is the definition of constrained Pareto optimality.

Consequently, the Drèze's prices do not depend only on the spot prices and the state prices of the stockholders. Nevertheless, we show that the present values of the firms with respect to these prices are consistent with the present values computed with the supporting prices of the consumers even if the state prices are not the same and the spot prices are not colinear. In particular, all present values coincide when short sales are allowed. This means that the evaluations of the stockholders coincide with the evaluation of the manager with the Drèze's prices.

To overcome the difficulty of defining an objective for the firms, we consider a pre-equilibrium, that is, a physical and financial feasible state such that the consumption plans are optimal in the budget sets. Then, if the preferences are smooth and if this allocation is constrained Pareto optimal in the weakest possible sense, we recover the expected formula for the firm's prices. Thus, in this case, we can define an objective for the firms, which is consistent with the optimality conditions, and, which is the natural extension of the Drèze's criterion.

For the proof, we use a non-convex separation theorem, which comes from an unpublished work of Cornet and Rockafellar, (see the proof in Aliprantis et al. (2002)). This result has been extended by Jofré and Rivera (1999) and we actually use a slight generalization of it to take into account the fact that our variables do not lie in the same linear space. It allows us to work with all variables avoiding partial approaches as in previous works.

The paper is organized as follows: in Section 2, we describe the general framework and the financial markets. Section 3 is devoted to the statements of the results with the necessary conditions for the constrained optimal allocations, the variants with different definitions, the consequences on the valuation of the production plans, and, finally, the extension of the Drèze's criterion. The last section gathers the mathematical arguments.

\section{Description of the economy}

We consider an inter-temporal economy with uncertainty. The model and the notations are essentially borrowed from Magill-Quinzii (1996). There are $T$ periods $(T \geq 2)$, and, $\mathcal{T}=\{0,1, \ldots, T\}$. As usual, the uncertainty is described by a tree of events (or nodes) $\mathbb{D}$. $\xi_{0}$ is the unique node at date $0 . \mathbb{D}_{t}$ denotes the nodes at date $t$. For each non-initial node $\xi \in \mathbb{D}^{+}=\mathbb{D} \backslash\left\{\xi_{0}\right\}$, there exists a unique predecessor denoted $\xi^{-}$. For each non-terminal node $\xi \in \mathbb{D}^{-}=\mathbb{D} \backslash \mathbb{D}_{T}$, we denote by $\xi^{+}$the set of immediate successors of $\xi$. Finally, we denote $\mathbb{D}_{0}^{-}=\mathbb{D}^{-} \backslash\left\{\xi_{0}\right\}$. We assume that there is $L$ perishable commodities at each node. Thus, the space of goods is $\mathbb{R}^{L \mathbb{D}}$. We denote by $\mathbf{1}$ the vector of $\mathbb{R}^{L}$, whose coordinates are all equal to 1 , and, for all $\xi \in \mathbb{D}$, by $\mathbf{1}_{\xi}$, the vector of $\mathbb{R}^{L \mathbb{D}}$ defined by $\mathbf{1}_{\xi}(\xi)=\mathbf{1}$ 
and $\mathbf{1}_{\xi}\left(\xi^{\prime}\right)=0$ for all $\xi^{\prime} \neq \xi$. Proj $\mathbf{1}^{\perp}$ denotes the orthogonal projection in $\mathbb{R}^{L}$ on $\mathbf{1}^{\perp}$ the orthogonal complement of $\mathbf{1}$.

A consumer/investor is represented by the superscript $i$ in the set $\mathcal{I}=\{1, \ldots, I\},(I \geq 1)$. A firm is represented by the superscript $j$ in the set $\mathcal{J}=\{1, \ldots, J\},(J \geq 1)$.

At each node, a market is open for the perishable commodities. For every non-terminal node, a stock market is open, on which the stocks of the firms are exchanged. The commodity prices are denoted by $p=(p(\xi))_{\xi \in \mathbb{D}} \in \mathbb{R}^{L \mathbb{D}}$. The share prices for the firm $j$ are denoted $q^{j}=\left(q^{j}(\xi)\right)_{\xi \in \mathbb{D}^{-}} \in \mathbb{R}^{\mathbb{D}^{-}}$. To simplify the notations, we also denote by $q^{j}$ the vector in $\mathbb{R}^{\mathbb{D}}$ where $q^{j}(\xi)=0$ for all $\xi \in \mathbb{D}_{T}$. A commodity bundle for the consumer $i$ is denoted $x^{i}=\left(x^{i}(\xi)\right)_{\xi \in \mathbb{D}} \in \mathbb{R}^{L \mathbb{D}}$. A portfolio is denoted $a^{i}=$ $\left(a_{j}^{i}(\xi)\right)_{\xi \in \mathbb{D}^{-}, j \in \mathcal{J}} \in\left(\mathbb{R}^{\mathbb{D}^{-}}\right)^{J}$. To simplify the notations, we also denote by $a^{i}$ the vector in $\left(\mathbb{R}^{\mathbb{D}}\right)^{J}$ where $a_{j}^{i}(\xi)=a_{j}^{i}\left(\xi^{-}\right)$for all $\xi \in \mathbb{D}_{T}$. The preferences of the $i^{\text {th }}$ agent is represented by the correspondence $P^{i}$ from the nonnegative orthant $\mathbb{R}_{+}^{L \mathbb{D}}$ to itself. For all $x^{i} \in \mathbb{R}_{+}^{L \mathbb{D}}, P^{i}\left(x^{i}\right)$ is the set of consumption plan strictly preferred by the agent to $x^{i}$. we posit the following weak monotony assumption at each state on the preferences. It means that the vector $\mathbf{1}$ is desirable in each state of nature.

Assumption C. For all $i \in \mathcal{I}$, for all $x^{i} \in \mathbb{R}_{+}^{\mathbb{D}}$ and for all $\xi \in \mathbb{D}$, there exists $\varepsilon>0$ such that for all $t \in] 0, \varepsilon\left[\right.$, for all $x^{i \prime} \in \bar{B}\left(x^{i}, \varepsilon\right) \cap \operatorname{adh} P^{i}\left(x^{i}\right), x^{i \prime}+t \mathbf{1}_{\xi} \in P^{i}\left(x^{i}\right)$.

We assume that every agent $i$ has an initial endowment of commodities denoted by $e^{i} \in \mathbb{R}_{+}^{L \mathbb{D}}$. At date 0 , the consumers have an initial share denoted for convenience $a^{i}\left(\xi_{0}^{-}\right)$. As usual, these shares satisfies the constraints $\sum_{i \in \mathcal{I}} a_{j}^{i}\left(\xi_{0}^{-}\right)=1$ for all $j \in \mathcal{J}$.

On the production side, every firm is described by a production set $Y^{j}$, a subset of $\mathbb{R}^{L \mathbb{D}}$. A production plan is denoted $y^{j}=\left(y^{j}(\xi)\right)_{\xi \in \mathbb{D}}$ and $y=\left(y^{j}\right)_{j \in \mathcal{J}} \in \prod_{j \in J} Y^{j}$ denotes a collections of production plans of the whole production sector. Let $y$ be a global production plan of the production sector, and, $(p, q)$ be commodity and stock prices. We now describe the budget constraints of agent $i$. At each node $\xi$, he receives the share of the profits (or losses) of the firms according to his portfolio at the previous node, he can trade on the commodity market at the price $p(\xi)$, and, at the non-terminal node, he can trade on the stock market at the price $q(\xi)$. Thus, the budget constraint is:

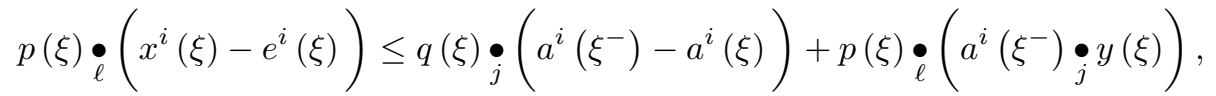

where $\bullet \ell$ denotes the standard inner product in $\mathbb{R}^{L}$, and, $\bullet_{j}$ denotes the standard inner product in $\mathbb{R}^{J}$, or, the sum over $j$, that is, $a^{i}(\xi) \bullet_{j} y(\xi)=\sum_{j \in \mathcal{J}} a_{j}^{i}(\xi) y^{j}(\xi)$. Thus, the budget set $B^{i}(p, q, y)$ of agent $i$ is:

$$
\left\{\begin{array}{l|l}
\left(x^{i}, a^{i}\right) \in \mathbb{R}_{+}^{L \mathbb{D}} \times\left(\mathbb{R}_{+}^{\mathbb{D}^{-}}\right)^{J} \mid \begin{array}{l}
p(\xi) \bullet \ell\left(x^{i}(\xi)-e^{i}(\xi)\right) \leq q(\xi) \bullet_{j}\left(a^{i}\left(\xi^{-}\right)-a^{i}(\xi)\right) \\
+p(\xi) \bullet \bullet_{\ell}\left(a^{i}\left(\xi^{-}\right) \bullet_{j} y(\xi)\right), \forall \xi \in \mathbb{D}
\end{array}
\end{array}\right\}
$$

If short sales are allowed, the only difference is that $a^{i}$ is chosen in $\left(\mathbb{R}^{\mathbb{D}^{-}}\right)^{J}$ instead of $\left(\mathbb{R}_{+}^{\mathbb{D}^{-}}\right)^{J}$.

\section{Statement of the Results}

Our aim is to provide a rule for the producers' behavior. We first recall the usual conditions for the consumers and for the markets at a stock market equilibrium. We call such collection a pre-equilibrium since we do not impose any restriction on the production plans.

Definition 1 A collections of prices and allocations $(p, q, x, a, y) \in \mathbb{R}^{L \mathbb{D}} \times\left(\mathbb{R}^{\mathbb{D}^{-}}\right)^{J} \times\left(\mathbb{R}_{+}^{L \mathbb{D}}\right)^{I} \times\left(\mathbb{R}_{+}^{\mathbb{D}^{-}}\right)^{I J} \times$ $\prod_{j \in \mathcal{J}} Y_{j}$ is a pre-equilibrium if

a) for each $i,\left(x^{i}, a^{i}\right) \in B^{i}(p, q, y)$, and $\left(P_{i}\left(x^{i}\right) \times\left(\mathbb{R}_{+}^{\mathbb{D}^{-}}\right)^{J}\right) \cap B^{i}(p, q, y)=\emptyset$; 
b) $\sum_{i \in \mathcal{I}}\left(x^{i}-e^{i}\right)=\sum_{j \in \mathcal{J}} y^{j}$;

c) for all $j \in \mathcal{J}$, for all $\xi \in \mathbb{D}^{-}, \sum_{i \in \mathcal{I}} a_{j}^{i}(\xi)=1$.

Remarks 1 The first condition means that the consumers choose an optimal consumption-portfolio pair taken the prices and the production plans as given. Condition b) requires that supply equals demand for every commodities at every node $\xi$, and, Condition c) requires the same for the stock markets.

The following result gives two consequences of Assumption $\mathbf{C}$ on the pre-equilibrium allocation. Its proof is a routine.

Proposition 1 Under Assumption $\mathbf{C}$, if $(p, q, x, a, y)$ is a pre-equilibrium, then for all states $\xi \in \mathbb{D}$, the budget constraint is binding, that is,

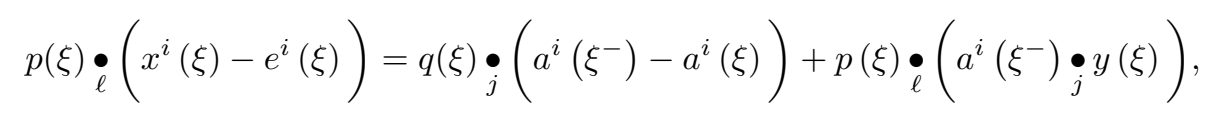

and the spot prices satisfy $p(\xi) \bullet \ell \mathbf{1}>0$ for all $\xi$.

Thus, from now on, we consider normalized spot prices in the affine hyperplan $H=\left\{p \in \mathbb{R}^{L} \mid\right.$ $\left.p \bullet_{\ell} \mathbf{1}=1\right\}$.

\subsection{Constrained Pareto-Optima}

This subsection is devoted to the problem of defining constrained attainable allocation with several goods and periods, and, to investigate the consequences on the necessary conditions of optimality and on the desirable behavior for the firms. The basic idea is: a constrained attainable allocation must be physically feasible in each node, the transfers among the consumers are free at the initial node, and, then, they must be financed through the stock market.

With one perishable commodity and two periods, there is only one-way to define what constrained feasibility means since it does not depend on the prices. With more than one commodity or more than two periods, the situation is quite different since, contrary to the usual Arrow-Debreu framework, the commodity prices and the asset prices matter. Hence, the definition may assume either that the prices are given and the transfers must be done through these prices, or, there exists some prices, which finance the transfers.

We consider the largest possible set of constrained allocation in this sub-section and in the following. Later, we discuss the modifications in the optimality conditions with weaker notions of constrained Pareto optimality. Note that we do not assume that the consumers are at an optimal consumption plan, according to Magill-Quinzii (Definition 31.7) (1996) but contrary to Geanakoplos et al (1990). Indeed, to be in the spirit of an Arrow-Debreu economy, we choose a concept of attainability, which is independent of consumers' preferences.

Definition 2 A collection of allocations $(x, a, y) \in\left(\mathbb{R}_{+}^{L \mathbb{D}}\right)^{I} \times\left(\left(\mathbb{R}_{+}^{\mathbb{D}^{-}}\right)^{J}\right)^{I} \times \prod_{j \in \mathcal{J}} Y_{j}$ is constrained feasible if :

a) there exist a stock price $q \in\left(\mathbb{R}^{\mathbb{D}^{+}}\right)^{J}$, with $q(\xi)=0$ for all $\xi \in \mathbb{D}_{T}$, and a commodity price $p \in H^{\mathbb{D}^{+}}$ such that for all $i \in \mathcal{I}$, for all $\xi \in \mathbb{D}^{+}$,

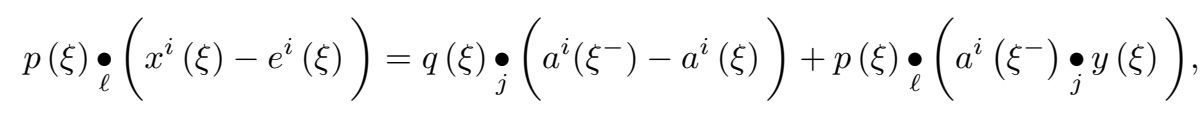

with $a^{i}(\xi)=a^{i}\left(\xi^{-}\right)$for all $\xi \in \mathbb{D}_{T} ;$

b) $\sum_{i \in \mathcal{I}}\left(x^{i}-e^{i}\right)=\sum_{j \in \mathcal{J}} y^{j}$; 
c) for all $j \in \mathcal{J}$, for all $\xi \in \mathbb{D}^{-}, \sum_{i \in \mathcal{I}} a_{j}^{i}(\xi)=1$.

The definition of constrained feasibility gives directly the one of constrained Pareto optimality.

Definition 3 A constrained feasible allocation $(x, a, y)$ is constrained Pareto optimal if there exists no Pareto superior constrained feasible allocation $\left(x^{\prime}, a^{\prime}, y^{\prime}\right)$ (i.e $x^{\prime i} \in P^{i}\left(x^{i}\right)$ for all $\left.i \in \mathcal{I}\right)$.

\subsection{First-Order Optimality Conditions of Constrained Efficiency}

The following result states the first order necessary condition for constrained Pareto optimal allocation. In the following, $N(C, c)$ denotes the Clarke's normal cone to $C$ at $c$, which coincides with the standard normal cone of convex analysis when $C$ is convex (See, Clarke (1983) for a precise definition and the main properties).

Theorem 3.1 Let $(\bar{x}, \bar{a}, \bar{y})$ be a constrained Pareto optimal allocation. Since it is constrained feasible, there exist a stock price vector $\bar{q} \in \mathbb{R}^{\mathbb{D}^{+}}$and a commodity price vector $\bar{p} \in H^{\mathbb{D}^{+}}$, which finance the allocation $(\bar{x}, \bar{a}, \bar{y})$. We assume that Assumption $\mathbf{C}$ holds true for each consumer, and, for all $j \in \mathcal{J}, Y^{j}$ is closed. Then, there exist $v \in\left(\mathbb{R}^{J}\right)^{\mathbb{D}^{-}}, p\left(\xi_{0}\right) \in \mathbb{R}^{L}$, with $p\left(\xi_{0}\right) \bullet \ell \mathbf{1} \geq 0, \sigma \in\left(\mathbf{1}^{\perp}\right)^{\mathbb{D}^{+}}$and $\pi \in\left(\mathbb{R}_{+}^{\mathbb{D}^{+}}\right)^{I}$, which satisfy the following conditions, with for all $i \in \mathcal{I}$,

$$
p^{i}=\left(p\left(\xi_{0}\right),\left(\pi^{i}(\xi) \bar{p}(\xi)+\sigma(\xi)\right)_{\xi \in \mathbb{D}^{+}}\right)
$$

and for all $j \in \mathcal{J}$,

$$
p^{j}=\left(p\left(\xi_{0}\right),\left(\sum_{i \in \mathcal{I}} a_{j}^{i}\left(\xi^{-}\right)\left(\pi^{i}(\xi) \bar{p}(\xi)+\sigma(\xi)\right)\right)_{\xi \in \mathbb{D}^{+}}\right) .
$$

(i) For all $i \in \mathcal{I}$,

$$
p^{i} \in-N\left(\operatorname{adh} P^{i}\left(\bar{x}^{i}\right), \bar{x}^{i}\right)
$$

and for some $i, p^{i} \neq 0$;

(ii) For all $j \in \mathcal{J}$,

$$
p^{j} \in N\left(Y^{j}, \bar{y}^{j}\right)
$$

(iii) For all $\xi \in \mathbb{D}^{+}$,

$$
\sum_{i \in \mathcal{I}} \pi^{i}(\xi)\left(\bar{x}^{i}(\xi)-e^{i}(\xi)-\sum_{j \in \mathcal{J}} \bar{a}_{j}^{i}\left(\xi^{-}\right) \bar{y}^{j}(\xi)\right)=0
$$

(iv) For all $\xi \in \mathbb{D}_{0}^{-}$,

$$
\sum_{i \in \mathcal{I}} \pi^{i}(\xi)\left(\bar{a}^{i}(\xi)-\bar{a}^{i}\left(\xi^{-}\right)\right)=0
$$

(v) For all $i \in \mathcal{I}, \forall \xi \in \mathbb{D}^{-}$,

$$
\begin{array}{r}
v(\xi)+\pi^{i}(\xi) \bar{q}(\xi)-\sum_{\xi^{\prime} \in \xi^{+}} \pi^{i}\left(\xi^{\prime}\right)\left(\bar{q}\left(\xi^{\prime}\right)+\left(\bar{p}\left(\xi^{\prime}\right) \bullet \bar{y}^{j}\left(\xi^{\prime}\right)\right)_{j \in \mathcal{J}}\right) \geq 0, \\
\bar{a}^{i}(\xi) \underset{j}{\bullet}\left(v(\xi)+\pi^{i}(\xi) \bar{q}(\xi)-\sum_{\xi^{\prime} \in \xi^{+}} \pi^{i}\left(\xi^{\prime}\right)\left(\bar{q}\left(\xi^{\prime}\right)+\left(\bar{p}\left(\xi^{\prime}\right) \bullet \bar{y}^{j}\left(\xi^{\prime}\right)\right)_{j \in \mathcal{J}}\right)\right)=0,
\end{array}
$$

with $\bar{q}\left(\xi_{0}\right)=0$. 
Condition $(i)$ means that the price $p^{i}$ is a supporting price for the consumption $\bar{x}^{i}$. In preferences are convex, this means that the consumer minimizes the expenditure at $\bar{x}^{i}$ for the price $p^{i}$. The presence of the term $\sigma(\xi)$ in the definition of the supporting price $p^{i}$ comes from the fact that, at each state of nature, the price, which finances the allocation, has no reason to be the price, which supports the allocation. With only one perishable commodity per state of nature, $\sigma(\xi)=0$ since the space of commodity at each state is one dimensional, and, thus the orthogonal to $\mathbf{1}$ is reduced to 0 . The presence of $\sigma$ in the formula defining $p^{i}$ implies that the supporting prices at each node are not necessarily colinear like in a standard Arrow-Debreu economy but stay in a two dimensional spaces. If the state prices $\pi^{i}$ are the same for the consumers, which is the case if the markets are complete for $(\bar{p}, \bar{q}, \bar{y})$, then we recover the equality of the supporting prices and they also coincide with the prices given in Condition (ii) for the producers.

Condition $(i i)$ is the basis for the definition of the Drèze's criterion. We come back at the end of the section on this point. Condition $(i i)$ means that the producers satisfy the first order necessary condition for profit maximization with respect to the price $p^{j}$. The price $p^{j}$ is computed from the supporting prices of the stockholders. At each node, $p^{j}(\xi)$ is a convex combination of the personal spot prices $p^{i}(\xi)$ of the stockholders and the coefficient are the shares at the previous node. This comes from the fact that the dividends are distributed according to the shares at the previous node. If we modify this convention, the formula will be changed according to the chosen dividend process.

Condition ( iii $)$ comes from the optimality condition with respect to spot prices $p(\xi)$. Indeed, in the definition of constrained feasibility, the planner has the possibility to choose the spot prices, which determine the possible transfers in the future. We show later that this condition disappear when we change the definition of constrained feasibility considering the spot prices as given. The same remark holds for Condition $(i v)$ and the stock prices $q^{j}$. With only one perishable commodity at each node, Condition (iii) is a consequence of the budget constraints and of Condition $(i v)$ since then $\bar{x}^{i}(\xi)-e^{i}(\xi)-\sum_{j \in \mathcal{J}} \bar{a}_{j}^{i}\left(\xi^{-}\right) \bar{y}^{j}(\xi)=q(\xi) \bullet_{j}\left(\bar{a}^{i}\left(\xi^{-}\right)-\left(\bar{a}^{i}(\xi)\right)\right.$. If all state prices are equal, then it is a consequence of the physical market clearing condition.

Under the same assumption, Condition $(i v)$ is a consequence of the market clearing condition on the stock market. In the general case, Conditions $(i i i)$ and $(i v)$ means that the weighted sums of the net trades either on the commodity market or on the stock market vanish in each state, taking the state prices as coefficients.

Condition $(v)$ corresponds to the absence of arbitrage opportunity for the consumer. Nevertheless, since the planner has the possibility to fix the stock prices, then, the conclusion is weaker than the usual one. Indeed, one gets an additional parameter $v(\xi)$, which is the same for all consumers. If the consumers are not constrained on the stock market, the usual condition of absence of arbitrage opportunity implies that $v(\xi)$ is equal to 0 .

In Bonnisseau-Lachiri (2002), we give an example of a constrained Pareto optimal allocation at which $v$ is not equal to 0 . This means that at such allocation, every consumers would like to sell (or to buy) the stocks for which $v^{j}(\xi)$ is different from 0 , but it is impossible at a global level since the market clearing condition would be violated. So, from an individual point of view, the allocation is not optimal, but from a global point of view, it is optimal.

If we allow for short sales, then Condition $(v)$ becomes stronger since equality holds true for every consumer and not only for the consumers having a positive share in the firm $j$. It suffices to remark that the normal cone computed in formula (17) of the proof is reduced to 0 since $\left(\mathbb{R}_{+}^{\mathbb{D}^{-}}\right)^{I}$ is replaced by $\left(\mathbb{R}^{\mathbb{D}^{-}}\right)^{I}$.

We now come back to the definition of constrained feasible allocation. Indeed, we may assume that either the stock price vector or the commodity price vector is fixed, or even that the two are given. This has a sense if we consider a pre-equilibrium where the price vectors are given and if we want to check if this allocation is optimal in some more or less strong sense. This means that the set of constrained feasible allocations is more or less large, and, consequently, being optimal is less demanding. Part of the conditions given in Theorem 3.1 remains true. The proof is easily deduced 
from the one given in the next section, since it means that some variables are kept fixed. Hence the computations are the same and even simpler, since they are less variables. More precisely, let us define three notions of weakly constrained Pareto optimal allocation as follows:

Definition 4 Let $(x, a, y) \in\left(\mathbb{R}_{+}^{L \mathbb{D}}\right)^{I} \times\left(\left(\mathbb{R}_{+}^{\mathbb{D}^{-}}\right)^{J}\right)^{I} \times \prod_{j \in \mathcal{J}} Y_{j}$ be a collection physically feasible, which means that it satisfies Conditions b) and c) of Definition 2.

(i) Let $p \in H^{\mathbb{D}^{+}}$. A collection $(x, a, y)$ is $p$-constrained feasible if there exists a stock price $q \in\left(\mathbb{R}^{\mathbb{D}^{+}}\right)^{J}$, with $q(\xi)=0$ for all $\xi \in \mathbb{D}_{T}$, such that for all $i \in \mathcal{I}$, for all $\xi \in \mathbb{D}^{+}$,

$$
p(\xi) \underset{\ell}{\bullet}\left(x^{i}(\xi)-e^{i}(\xi)\right)=q(\xi) \underset{j}{\bullet}\left(a^{i}\left(\xi^{-}\right)-a^{i}(\xi)\right)+p(\xi) \underset{\ell}{\bullet}\left(a^{i}\left(\xi^{-}\right) \underset{j}{\bullet} y(\xi)\right),
$$

with $a^{i}(\xi)=a^{i}\left(\xi^{-}\right)$for all $\xi \in \mathbb{D}_{T}$;

(ii) Let $q \in\left(\mathbb{R}^{\mathbb{D}^{+}}\right)^{J}$, with $q(\xi)=0$ for all $\xi \in \mathbb{D}_{T}$. A collection $(x, a, y)$ is $q$-constrained feasible if there exists $p \in H^{\mathbb{D}^{+}}$such that for all $i \in \mathcal{I}$, for all $\xi \in \mathbb{D}^{+}$,

$$
p(\xi) \underset{\ell}{\bullet}\left(x^{i}(\xi)-e^{i}(\xi)\right)=q(\xi) \underset{j}{\bullet}\left(a^{i}\left(\xi^{-}\right)-a^{i}(\xi)\right)+p(\xi) \underset{\ell}{\bullet}\left(a^{i}\left(\xi^{-}\right) \underset{j}{\bullet} y(\xi)\right),
$$

with $a^{i}(\xi)=a^{i}\left(\xi^{-}\right)$for all $\xi \in \mathbb{D}_{T}$;

(iii) Let $p \in H^{\mathbb{D}^{+}}$and $q \in\left(\mathbb{R}^{\mathbb{D}^{+}}\right)^{J}$, with $q(\xi)=0$ for all $\xi \in \mathbb{D}_{T}$. A collection $(x, a, y)$ is $(p, q)$ constrained feasible if for all $i \in \mathcal{I}$, for all $\xi \in \mathbb{D}^{+}$,

$$
p(\xi) \underset{\ell}{\bullet}\left(x^{i}(\xi)-e^{i}(\xi)\right)=q(\xi) \underset{j}{\bullet}\left(a^{i}\left(\xi^{-}\right)-a^{i}(\xi)\right)+p(\xi) \underset{\ell}{\bullet}\left(a^{i}\left(\xi^{-}\right) \underset{j}{\bullet} y(\xi)\right),
$$

with $a^{i}(\xi)=a^{i}\left(\xi^{-}\right)$for all $\xi \in \mathbb{D}_{T}$;

A $p$-constrained (resp. q-constrained, resp. $(p, q)$-constrained) feasible allocation $(x, a, y)$ is weakly $p$-constrained (resp. $q$-constrained, resp. $(p, q)$-constrained) Pareto optimal if there exists no Pareto superior p-constrained (resp. q-constrained, resp. ( $p, q)$-constrained) feasible allocation.

Corollary 3.1 Let $(\bar{x}, \bar{a}, \bar{y})$ be a weakly p-constrained (resp. q-constrained, resp. (p,q)-constrained) Pareto optimal allocation. Under the assumptions of Theorem 3.1 and with their notations, Conditions (i), (ii), (iv) and (v) (resp. (i), (ii), (iii) and (v), resp. (i), (ii) and (v)) hold true.

Note that the Conditions given by the corollary are less restrictive than the one given by Theorem 3.1 , which is a consequence of the fact that the set of feasible allocations is smaller. Nevertheless, the two first conditions always hold true, which means that the supporting prices for the consumptions and for the productions remain the same whatever is the definition of constrained feasibility.

\subsection{On the value of the firms}

In the following proposition, we compare the present values of the production plan evaluated at the price $p^{j}$ and at the personal price $p^{i}$ for each consumer. If Conditions $(i v)$ and $(v)$ of Theorem 3.1 hold, then equality holds if the consumer is a stockholder at each state or if short sales are allowed. So even if the stockholders do not have the same personal prices, even if these prices in a state are not colinear, it remains that the present value of a production plan is the same for all consumers and equal to the present value computed with the supporting price of the firm. Thus, the present value of a production plan is well defined if the necessary conditions for constrained Pareto optimality are satisfied. Consequently, if the manager of the firm is asked to choose a production plan according to Condition ( $\mathrm{ii}$ ), then her/his evaluation of the present value of the firm is equal to the one of the stockholders. If short sales are not allowed, one only gets an inequality, that is the evaluation by 
the price $p^{j}$ is greater or equal to the evaluation by the price $p^{i}$. Note that the shareholders does not receive a part of the profit at the states where her/his share is 0 . Thus, she/he is interested to compute the present value taking into account only the states at which she/he has a positive share. Then, the partial evaluations coincide if we restrict the computation to those states.

Proposition 2 Let $(\bar{x}, \bar{a}, \bar{y})$ be a constrained feasible allocation. Let $\bar{q} \in \mathbb{R}^{\mathbb{D}_{0}^{-}}$a stock price and $\bar{p} \in$ $H^{\mathbb{D}^{+}}$, which finance it. If the necessary conditions $(i v)$ and $(v)$ of Theorem 3.1 are satisfied, then, with the notations of Theorem 3.1, one has for all $j \in \mathcal{J}$, for all $i \in \mathcal{I}$,

$$
\Pi^{j}=p^{j} \cdot \bar{y}^{j}=p\left(\xi_{0}\right) \bullet \bar{y}^{j}\left(\xi_{0}\right)+\sum_{\xi \in \mathbb{D}^{-}} v^{j}(\xi)+\sum_{\xi \in \mathbb{D}^{+}} \sigma(\xi) \bullet \bar{y}^{j}(\xi) \geq p^{i} \cdot \bar{y}^{j} .
$$

There is an equality if $\bar{a}_{j}^{i}(\xi)>0$ for all $\xi \in \mathbb{D}^{-}$or if short sales are allowed.

Proof.

$$
\begin{aligned}
\Pi^{j} & =p\left(\xi_{0}\right) \bullet \bar{y}^{j}\left(\xi_{0}\right)+\sum_{\xi \in \mathbb{D}^{+}} \sum_{i \in \mathcal{I}} \bar{a}_{j}^{i}\left(\xi^{-}\right)\left(\pi^{i}(\xi) \bar{p}(\xi)+\sigma(\xi)\right) \bullet \bar{y}_{\ell}^{j}(\xi) \\
& =p\left(\xi_{0}\right) \bullet \bar{y}^{j}\left(\xi_{0}\right)+\sum_{t=1}^{T} \sum_{\xi \in \mathbb{D}_{t}} \sum_{i \in \mathcal{I}} \pi^{i}(\xi) \bar{a}_{j}^{i}\left(\xi^{-}\right) \bar{p}(\xi) \bullet \bar{y}^{j}(\xi)+\sum_{\xi \in \mathbb{D}^{+}} \sigma(\xi) \bullet \bar{y}^{j}(\xi) .
\end{aligned}
$$

The equality comes from the fact that $\sum_{i \in \mathcal{I}} \bar{a}_{j}^{i}\left(\xi^{-}\right)=1$ for all $\xi$. For each $t$ between 0 and $T-1$, summing Equalities (6) of Condition $(v)$ with respect to $\xi \in \mathbb{D}_{t}$ and to $i \in \mathcal{I}$, one obtains

$$
\begin{aligned}
\sum_{\xi^{\prime} \in \mathbb{D}_{t+1}} \sum_{i \in \mathcal{I}} \bar{a}_{j}^{i}\left(\xi^{\prime}\right) \pi^{i}\left(\xi^{\prime}\right) \bar{p}\left(\xi^{\prime}\right) \bar{\ell}_{\ell} \bar{y}^{j}\left(\xi^{\prime}\right) & =\sum_{\xi \in \mathbb{D}_{t}} \sum_{i \in \mathcal{I}} \bar{a}_{j}^{i}(\xi)\left(v^{j}(\xi)+\pi^{i}(\xi) \bar{q}^{j}(\xi)\right) \\
& -\sum_{\xi^{\prime} \in \mathbb{D}_{t+1}} \sum_{i \in \mathcal{I}} \bar{a}_{j}^{i}\left(\xi^{\prime-}\right) \pi^{i}\left(\xi^{\prime}\right) \bar{q}^{j}\left(\xi^{\prime}\right),
\end{aligned}
$$

with $\bar{q}^{j}(\xi)=0$ for all $\xi \in \mathbb{D}_{T}$. Since $\sum_{i \in \mathcal{I}} \bar{a}_{j}^{i}(\xi)=1$, one has

$$
\sum_{\xi \in \mathbb{D}_{t}} \sum_{i \in \mathcal{I}} \bar{a}_{j}^{i}(\xi) v^{j}(\xi)=\sum_{\xi \in \mathbb{D}_{t}} v^{j}(\xi) .
$$

From condition $(i v)$, for all $t \geq 1$, one has

$$
\sum_{\xi \in \mathbb{D}_{t}} \sum_{i \in \mathcal{I}} \bar{a}_{j}^{i}(\xi) \pi^{i}(\xi) \bar{q}^{j}(\xi)=\sum_{\xi \in \mathbb{D}_{t}} \sum_{i \in \mathcal{I}} \bar{a}_{j}^{i}\left(\xi^{-}\right) \pi^{i}(\xi) \bar{q}^{j}(\xi) .
$$

Consequently, one gets, for all $t \geq 1$,

$$
\begin{aligned}
\sum_{\xi^{\prime} \in \mathbb{D}_{t+1}} \sum_{i \in \mathcal{I}} \bar{a}_{j}^{i}\left(\xi^{\prime-}\right) \pi^{i}\left(\xi^{\prime}\right) \bar{p}\left(\xi^{\prime}\right) \bullet \bar{y}^{j}\left(\xi^{\prime}\right)= & \sum_{\xi \in \mathbb{D}_{t}} v^{j}(\xi)+\sum_{\xi \in \mathbb{D}_{t}} \sum_{i \in \mathcal{I}} \bar{a}_{j}^{i}\left(\xi^{-}\right) \pi^{i}(\xi) \bar{q}^{j}(\xi) \\
& -\sum_{\xi^{\prime} \in \mathbb{D}_{t+1}} \sum_{i \in \mathcal{I}} \bar{a}_{j}^{i}\left(\xi^{\prime-}\right) \pi^{i}\left(\xi^{\prime}\right) \bar{q}^{j}\left(\xi^{\prime}\right),
\end{aligned}
$$

and,

$$
\sum_{\xi^{\prime} \in \mathbb{D}_{1}} \sum_{i \in \mathcal{I}} \bar{a}_{j}^{i}\left(\xi^{\prime-}\right) \pi^{i}\left(\xi^{\prime}\right) \bar{p}\left(\xi^{\prime}\right) \bullet \bar{y}^{j}\left(\xi^{\prime}\right)=v^{j}\left(\xi_{0}\right)-\sum_{\xi^{\prime} \in \mathbb{D}_{1}} \sum_{i \in \mathcal{I}} \bar{a}_{j}^{i}\left(\xi^{\prime-}\right) \pi^{i}\left(\xi^{\prime}\right) \bar{q}^{j}\left(\xi^{\prime}\right) .
$$


Since $\bar{q}(\xi)=0$ for all $\xi \in \mathbb{D}_{T}$, summing over $t$, one gets,

$$
\sum_{t=1}^{T} \sum_{\xi \in \mathbb{D}_{t}} \sum_{i \in \mathcal{I}} \pi^{i}(\xi) \bar{a}_{j}^{i}\left(\xi^{-}\right) \bar{p}(\xi) \bullet \bar{y}^{j}(\xi)=\sum_{\xi \in \mathbb{D}^{-}} v^{j}(\xi)
$$

Consequently, this leads to the announced formula. In the same way, one computes $p^{i} \cdot \bar{y}^{j}$, but one has to replace some equalities by inequalities at the states $\xi$ such that $\bar{a}_{j}^{i}(\xi)=0$, which gives the inequality $p^{j} \cdot \bar{y}^{j} \geq p^{i} \cdot \bar{y}^{j}$. When short sales are possible or when a consumer is a stockholder of the firm at each node, there are only equalities, hence, one gets also an equality after the computation.

\subsection{On the objective of the firm}

We present in this subsection the link with the Drèze's criterion (1974) extended in Bonnisseau-Lachiri (2002) to a multi-period economy with only one commodity per state. Indeed, the Drèze's criterion can be defined as follows: the firms must maximize the profit (or at least satisfy a necessary condition for profit maximization) with respect to the Drèze's prices $\left(p^{j}\right)$ defined by: for every $j \in \mathcal{J}$,

$$
p^{j}(\xi)= \begin{cases}p\left(\xi_{0}\right) & \text { if } \xi=\xi_{0} \\ \left(\sum_{i \in \mathcal{I}} \pi^{i}(\xi) \cdot \bar{a}_{j}^{i}\left(\xi^{-}\right)\right) \cdot \bar{p}(\xi) & \text { if } \xi \in \mathbb{D}^{+}\end{cases}
$$

for all $\xi \in \mathbb{D}^{+}$.

This price is obtained by considering state prices, which are a convex combination of the state prices of the stockholders, where the weights are the shares at the previous node. Nevertheless, the Condition $(\mathrm{ii})$ of Theorem 3.1 does not fit with this definition since one has this additional parameter $\sigma(\xi)$. The following proposition shows that we recover the Drèze's price at a constrained Pareto optimal allocation, when it is also a pre-equilibrium and the preferences are smooth.

Proposition 3 Let $(\bar{p}, \bar{q}, \bar{x}, \bar{a}, \bar{y})$ be a weakly $(\bar{p}, \bar{q})$-constrained Pareto optimal pre-equilibrium with $\bar{p} \in H^{\mathbb{D}}$. Under the Assumption of Theorem 3.1, if for each consumer, $N\left(\operatorname{adh} P^{i}\left(\bar{x}^{i}\right), \bar{x}^{i}\right)$ is an half line, then there exists $p\left(\xi_{0}\right) \in \mathbb{R}^{L}$ and $\pi \in\left(\mathbb{R}_{+}^{\mathbb{D}^{+}}\right)^{I}$, such that Conditions (i), (ii) and (v) of Theorem 3.1 hold true with $\sigma=0, v=0$ and, either $p\left(\xi_{0}\right)=\bar{p}\left(\xi_{0}\right)$, or $p\left(\xi_{0}\right)=0$.

Proof of Proposition 3. We start by recalling the necessary conditions for the consumer's maximization problem.

Lemma 1 Let us assume that the preferences satisfy Assumption $\mathbf{C}$. Let $p \in H^{\mathbb{D}}, q \in \mathbb{R}^{-}$and $y \in \prod_{j \in \mathcal{J}} Y^{j}$. If $\left(\bar{x}^{i}, \bar{a}^{i}\right) \in \mathbb{R}_{+}^{L \mathbb{D}} \times\left(\mathbb{R}_{+}^{\mathbb{D}^{-}}\right)^{J}$ is a maximal element of $P^{i}$ in the budget set $B^{i}(p, q, y)$, then, with $q(\xi)=0$ for all $\xi \in \mathbb{D}_{T}$,

$$
p(\xi) \underset{\ell}{\bullet}\left(\bar{x}^{i}(\xi)-e^{i}(\xi)\right)=q(\xi) \underset{j}{\bullet}\left(a^{i}\left(\xi^{-}\right)-a^{i}(\xi)\right)+p(\xi) \underset{\ell}{\bullet}\left(a^{i}\left(\xi^{-}\right) \underset{j}{\bullet} y(\xi)\right), \forall \xi \in \mathbb{D} ;
$$

and there exists $\pi^{i} \in \mathbb{R}_{+}^{\mathbb{D}} \backslash\{0\}$ such that

$$
-\left(\pi^{i}(\xi) p(\xi)\right)_{\xi \in \mathbb{D}} \in N\left(\operatorname{adh} P^{i}\left(\bar{x}^{i}\right), \bar{x}^{i}\right) ;
$$

$\forall \xi \in \mathbb{D}^{-}$,

$$
\begin{array}{r}
\pi^{i}(\xi) q(\xi)-\sum_{\xi^{\prime} \in \xi^{+}} \pi^{i}\left(\xi^{\prime}\right)\left(q\left(\xi^{\prime}\right)+\left(p\left(\xi^{\prime}\right) \bullet y_{\ell} y^{j}\left(\xi^{\prime}\right)\right)_{j \in \mathcal{J}}\right) \geq 0, \\
\bar{a}^{i}(\xi) \underset{j}{\bullet}\left(\pi^{i}(\xi) q(\xi)-\sum_{\xi^{\prime} \in \xi^{+}} \pi^{i}\left(\xi^{\prime}\right)\left(q\left(\xi^{\prime}\right)+\left(p\left(\xi^{\prime}\right)_{\ell} y^{j}\left(\xi^{\prime}\right)\right)_{j \in \mathcal{J}}\right)\right)=0 .
\end{array}
$$


Conversely, if $\left(\bar{x}^{i}, \bar{a}^{i}\right) \in\left(\mathbb{R}_{+}^{L}\right)^{\mathbb{D}} \times\left(\mathbb{R}_{+}^{\mathbb{D}^{-}}\right)^{J}$ satisfies the above conditions with respect to $\pi^{i} \in \mathbb{R}_{+}^{\mathbb{D}}$, if $\sum_{\xi \in \mathbb{D}} \pi^{i}(\xi) p(\xi) \bullet \bar{x}^{i}(\xi)>0$, and if $P^{i}\left(\bar{x}_{i}\right)$ is open and convex, then $\left(\bar{x}^{i}, \bar{a}^{i}\right)$ is a maximal element of $P^{i}$ in the budget set and $\pi^{i} \in \mathbb{R}_{++}^{\mathbb{D}}$.

The proof of this lemma is given in the next section.

We now apply Corollary 3.1 to $(\bar{x}, \bar{a}, \bar{y})$, which is financed by the prices $\bar{p}$ and $\bar{q}$. There exist $v \in\left(\mathbb{R}^{J}\right)^{\mathbb{D}^{-}}, p\left(\xi_{0}\right) \in \mathbb{R}^{L}, \sigma \in\left(\mathbf{1}^{\perp}\right)^{\mathbb{D}^{+}}$and $\pi \in\left(\mathbb{R}_{+}^{I}\right)^{\mathbb{D}^{+}}$, which satisfy Conditions $(i),(i i)$ and $(v)$ of Theorem 3.1. From Lemma 1, for all $i$, there exists $\pi^{i \prime} \in \mathbb{R}_{+}^{\mathbb{D}} \backslash\{0\}$ such that $-\left(\pi^{i \prime}(\xi) \bar{p}(\xi)\right)_{\xi \in \mathbb{D}} \in$ $N\left(\operatorname{adh} P^{i}\left(\bar{x}^{i}\right), \bar{x}^{i}\right)$. Since the preferences are smooth, the vectors $p^{i}=\left(p\left(\xi_{0}\right),\left(\pi^{i}(\xi) \bar{p}(\xi)+\sigma(\xi)\right)\right)$ and $\left(\pi^{i \prime}(\xi) \bar{p}(\xi)\right)$ are positively colinear. Since at least one price $p^{i}$ is not equal to 0 and $\pi^{i \prime}$ is different from 0 , one gets that there exists $t^{i}>0$ such that $t^{i} \pi^{i \prime}\left(\xi_{0}\right) \bar{p}\left(\xi_{0}\right)=p\left(\xi_{0}\right)$ ) and, for all $\xi \in \mathbb{D}^{+}$, $t^{i} \pi^{i \prime}(\xi) \bar{p}(\xi)=\pi^{i}(\xi) \bar{p}(\xi)+\sigma(\xi)$. Since $\bar{p}(\xi) \in H$, one deduces that $\sigma(\xi)=0$. If $\pi^{i \prime}\left(\xi_{0}\right)>0, p\left(\xi_{0}\right)$ is positively proportional to $\bar{p}\left(\xi_{0}\right)$, and, since the $p\left(\xi_{0}\right)$ and $\pi$ are defined up to the multiplication by a positive scalar, we can choose $p\left(\xi_{0}\right)=\bar{p}\left(\xi_{0}\right)$. Finally, for each $i \in \mathcal{I}$, either $\pi^{i}$ is positively proportional to $\pi^{i \prime}$ or is equal to 0 . Then Condition (9) of Lemma 1 implies that $v=0$ in Condition $(v)$ of Theorem 3.1 since at each node, there exists at least one consumer such that $\bar{a}_{j}^{i}(\xi)>0$.

We can conclude with the following definition of a stock market equilibrium in which the list of conditions incorporate the one for a pre-equilibrium and an additional one for the producers: the Drèze's criterion. The firms are asked to choose a production plan, which satisfies the first order necessary condition for profit maximization with respect to the Drèze's price given by Condition (ii) of Theorem 3.1. The formulation is intricate since the state prices of the consumers enter in the definition of the Drèze's price.

Definition 5 A collections of prices and allocations $(p, q, x, a, y) \in \mathbb{R}^{L \mathbb{D}} \times\left(\mathbb{R}^{\mathbb{D}^{-}}\right)^{J} \times\left(\mathbb{R}_{+}^{L \mathbb{D}}\right)^{I} \times\left(\mathbb{R}_{+}^{\mathbb{D}^{-}}\right)^{I J} \times$ $\prod_{j \in \mathcal{J}} Y_{j}$ is a stock market equilibrium if it is a pre-equilibrium, and, if there exist $\pi\left(\xi_{0}\right) \geq 0, \pi \in\left(\mathbb{R}_{+}^{\mathbb{D}^{+}}\right)^{I}$ such that for all $i \in \mathcal{I}, 0 \neq\left(\pi\left(\xi_{0}\right),\left(\pi^{i}(\xi)\right)\right)$ and Conditions (8) and (9) of Lemma 1 hold true, and, for all $j \in \mathcal{J}$,

$$
p^{j}=\left(\pi\left(\xi_{0}\right) p\left(\xi_{0}\right),\left(\left(\sum_{i \in \mathcal{I}} \pi^{i}(\xi) a_{j}^{i}\left(\xi^{-}\right)\right) p(\xi)\right)\right) \in N\left(Y^{j}, y^{j}\right) .
$$

\section{Proofs of the results}

\subsection{Proof of Lemma 1.}

First step: necessary conditions. If $\left(\bar{x}^{i}, \bar{a}^{i}\right)$ is a maximal element, one has

$$
\left(\bar{x}^{i}, \bar{a}^{i}\right) \in B^{i}(p, q, y) \text { and } \tilde{P}^{i}\left(\bar{x}^{i}\right) \cap B^{i}(p, q, y)=\emptyset,
$$

with

$$
\tilde{P}^{i}\left(\bar{x}^{i}\right)=P^{i}\left(\bar{x}^{i}\right) \times\left(\mathbb{R}^{\mathbb{D}^{-}}\right)^{J}
$$

and $B^{i}(p, q, y)$ is equal to

$$
\left\{\begin{array}{l|l}
\left(x^{i}, a^{i}\right) \in\left(\mathbb{R}_{+}^{L}\right)^{\mathbb{D}} \times\left(\mathbb{R}_{+}^{\mathbb{D}^{-}}\right)^{J} \mid \begin{array}{l}
p(\xi) \bullet_{\ell}\left(x^{i}(\xi)-e^{i}(\xi)\right) \leq q(\xi) \bullet_{j}\left(a^{i}\left(\xi^{-}\right)-a^{i}(\xi)\right) \\
+p(\xi) \bullet_{\ell}\left(a^{i}\left(\xi^{-}\right) \bullet_{j} y(\xi)\right), \forall \xi \in \mathbb{D}
\end{array}
\end{array}\right\}
$$

As already noticed, Condition (7) holds true due to Assumption C. To find the other necessary conditions, we apply the following result of Cornet-Rockafellar (see the proof in Aliprantis et al. (2002)). 
Proposition 4 Let $\left(C_{\kappa}\right)_{\kappa=1, \ldots, k}, k$ closed subsets of a finite dimensional Euclidean space. Let $\left(c_{\kappa}\right) \in$ $\prod_{\kappa=1}^{k} C_{\kappa}$ such that $\sum_{\kappa=1}^{k} c_{\kappa} \in \operatorname{Fr}\left(\sum_{\kappa=1}^{k} C_{\kappa}\right)$. Then, $\cap_{\kappa=1}^{k} N\left(C_{\kappa}, c_{\kappa}\right) \neq\{0\}$.

From Assumption $\mathbf{C}$, there exists $\varepsilon>0$ such that for all $t \in] 0, \varepsilon\left[\right.$, for all $x^{i} \in B\left(\bar{x}^{i}, \varepsilon\right) \cap \operatorname{adh} P^{i}\left(\bar{x}^{i}\right)$, $x^{i}+t \mathbf{1}_{\xi_{0}} \in P^{i}\left(\bar{x}^{i}\right)$. Let $C_{1}=B^{i}(p, q, y), C_{2}=\left(B\left(\bar{x}^{i}, \varepsilon\right) \cap \operatorname{adh} P^{i}\left(\bar{x}^{i}\right)\right) \times\left(\mathbb{R}^{\mathbb{D}^{-}}\right)^{J}$ and $c_{1}=c_{2}=\left(\bar{x}^{i}, \bar{a}^{i}\right)$. We now show that $c_{1}-c_{2}=0$ belongs to the boundary of $C_{1}-C_{2}$. Let $\mathbf{v}=\left(\mathbf{1}_{\xi_{0}}, 0\right) \in\left(\mathbb{R}^{L}\right)^{\mathbb{D}} \times\left(\mathbb{R}^{\mathbb{D}^{-}}\right)^{J}$. It suffices to show that, for all $t \in] 0, \varepsilon\left[, t \mathbf{v} \notin C_{1}-C_{2}\right.$. If it is not true, there exist $\left.t \in\right] 0, \varepsilon\left[,\left(\tilde{x}^{i}, \tilde{a}^{i}\right) \in\right.$ $\left(B\left(\bar{x}^{i}, \varepsilon\right) \cap \operatorname{adh} P^{i}\left(\bar{x}^{i}\right)\right) \times\left(\mathbb{R}^{\mathbb{D}^{-}}\right)^{J}$ and $\left(x^{i}, a^{i}\right) \in B^{i}(p, q, y)$ such that $x^{i}=\tilde{x}^{i}+t \mathbf{1}_{\xi_{0}}$ and $a^{i}=\tilde{a}^{i}$. From Assumption $\mathbf{C}, x^{i}=\tilde{x}^{i}+t \mathbf{1}_{\xi_{0}} \in P^{i}\left(\bar{x}^{i}\right)$. Thus $\left(x^{i}, a^{i}\right) \in \tilde{P}^{i}\left(\bar{x}^{i}\right)$. Since, one also has $\left(x^{i}, a^{i}\right) \in B^{i}(p, q, y)$, this contradicts $\tilde{P}^{i}\left(\bar{x}^{i}\right) \cap B^{i}(p, q, y)=\emptyset$.

We now apply the previous proposition. Thus there exists a vector $\left(\alpha^{i}, \varpi^{i}\right) \in \mathbb{R}^{\mathbb{D}} \times\left(\mathbb{R}^{\mathbb{D}^{-}}\right)^{J},\left(\alpha^{i}, \varpi^{i}\right) \neq$ 0 such that

$$
\left(\alpha^{i}, \varpi^{i}\right) \in N\left(-C_{2},-\left(\bar{x}^{i}, \bar{a}^{i}\right)\right)
$$

and

$$
\left(\alpha^{i}, \varpi^{i}\right) \in N\left(B^{i}(p, q, y),\left(\bar{x}^{i}, \bar{a}^{i}\right)\right)
$$

From the properties of the Clarke's normal cone (see, Clarke (1983)), one has

$$
\begin{aligned}
N\left(-C_{2},-\left(\bar{x}^{i}, \bar{a}^{i}\right)\right) & =-N\left(\operatorname{adh} P^{i}\left(\bar{x}^{i}\right), \bar{x}^{i}\right) \times-N\left(\left(\mathbb{R}^{\mathbb{D}^{-}}\right)^{J}, \bar{a}^{i}\right) \\
& =N\left(\operatorname{adh} P\left(\bar{x}^{i}\right), \bar{x}^{i}\right) \times\left\{0_{\mathbb{D}}\right\}^{J} .
\end{aligned}
$$

Since $B^{i}(p, q, y)$ is a closed convex subset, Clarke's normal cone is the standard cone of the convex analysis. Thus, $\left(\alpha^{i}, \varpi^{i}\right) \in N\left(B^{i}(p, q, y),\left(\bar{x}^{i}, \bar{a}^{i}\right)\right)$ if there exist $\vartheta^{i} \in\left(\mathbb{R}_{+}^{L}\right)^{\mathbb{D}}, \tau_{j}^{i} \in\left(\mathbb{R}_{+}^{\mathbb{D}^{-}}\right)^{J}$ and $\varsigma^{i} \in \mathbb{R}_{+}^{\mathbb{D}}$ such that

1. $\alpha^{i}(\xi)=\varsigma^{i}(\xi) p(\xi)-\vartheta^{i}(\xi), \forall \xi \in \mathbb{D}$;

2. $\varpi_{j}^{i}(\xi)=-\tau_{j}^{i}(\xi)+\varsigma^{i}(\xi) q^{j}(\xi)-\sum_{\xi^{\prime} \in \xi^{+}} \varsigma^{i}\left(\xi^{\prime}\right)\left(q^{j}\left(\xi^{\prime}\right)+y^{j}\left(\xi^{\prime}\right)\right), \forall \xi \in \mathbb{D}^{-}$;

3. $\vartheta^{i}(\xi) \bullet{ }_{\ell} \bar{x}^{i}(\xi)=0, \forall \xi \in \mathbb{D}$.

4. $\tau_{j}^{i}(\xi) \bar{a}_{j}^{i}(\xi)=0, \forall j \in \mathcal{J}, \forall \xi \in \mathbb{D}^{-}$.

Relations (10) and (11) together with the computations of the normal cone lead to:

$$
-\alpha^{i}=\left(\vartheta^{i}-\varsigma^{i}\right) \in N\left(\operatorname{adh} P^{i}\left(\bar{x}^{i}\right), \bar{x}^{i}\right) \text { and } \varpi^{i}=0
$$

and

$$
\text { for every } j \in \mathcal{J}, \tau_{j}^{i}(\xi)=\varsigma^{i}(\xi) q^{j}(\xi)-\sum_{\xi^{\prime} \in \xi^{+}} \varsigma^{i}\left(\xi^{\prime}\right)\left(q^{j}\left(\xi^{\prime}\right)+y^{j}\left(\xi^{\prime}\right)\right), \forall \xi \in \mathbb{D}^{-} \text {. }
$$

Equation (9) of Lemma 1 is deduced from the above equations and relations 2 and 4 with $\pi^{i}=\varsigma^{i}$. We now show that $-\left(\pi^{i}(\xi) p(\xi)\right)$ belongs to $N\left(\operatorname{adh} P^{i}\left(\bar{x}^{i}\right), \bar{x}^{i}\right)$. Since $-\left(\pi^{i}(\xi) p(\xi)\right)_{\xi}=-\alpha^{i}-\vartheta^{i}$, since $N\left(\operatorname{adh} P^{i}\left(\bar{x}^{i}\right), \bar{x}^{i}\right)$ is a convex cone and $-\alpha^{i}$ belongs to it, it suffices to show that $-\vartheta^{i}$ belongs to it. Note that $\vartheta^{i} \in\left(\mathbb{R}_{+}^{L}\right)^{\mathbb{D}}$ and $\vartheta^{i}(\xi) \bullet \bar{x}^{i}(\xi)=0$. Consequently, the projection of $\bar{x}^{i}-\vartheta^{i}$ on $\left(\mathbb{R}_{+}^{L}\right)^{\mathbb{D}}$ is $\bar{x}^{i}$. Hence, since $\operatorname{adh} P^{i}\left(\bar{x}^{i}\right) \subset \mathbb{R}_{+}^{\mathbb{D}}$, the projection of $\bar{x}^{i}-\vartheta^{i}$ on $\operatorname{adh} P^{i}\left(\bar{x}^{i}\right)$ is $\bar{x}^{i}$. This means that 
$-\vartheta^{i}$ is perpendicular to $\operatorname{adh} P^{i}\left(\bar{x}^{i}\right)$ at $\bar{x}^{i}$ and thus it belongs to $N\left(\operatorname{adh} P^{i}\left(\bar{x}^{i}\right), \bar{x}^{i}\right)(\operatorname{See}, \operatorname{Clarke}(1983))$. We now prove that $\pi^{i} \neq 0$. If it is not true, then the relation 1 implies that $\alpha^{i} \leq 0$. Furthermore, $\alpha^{i} \neq 0$ since $\left(\alpha^{i}, \varpi^{i}\right) \neq 0$ and $\varpi^{i}=0$. From Assumption $\mathbf{C}$, for all $\xi \in \mathbb{D}, \mathbf{1}_{\xi}$ belongs to the polar of the normal cone $N\left(\operatorname{adh} P^{i}\left(\bar{x}^{i}\right), \bar{x}^{i}\right)$ that is $T\left(\operatorname{adh} P^{i}\left(\bar{x}^{i}\right), \bar{x}^{i}\right)$. Then, since $\alpha^{i} \in-N\left(\operatorname{adh} P^{i}\left(\bar{x}^{i}\right), \bar{x}^{i}\right)$, one deduces that $\alpha^{i} \cdot \mathbf{1}_{\xi}=\alpha^{i}(\xi) \bullet \mathbf{1} \geq 0$, which together with $\alpha^{i} \leq 0$ implies $\alpha^{i}=0$. Hence, one gets a contradiction.

Second step: sufficient conditions. Let $\left(\bar{x}^{i}, \bar{a}^{i}\right) \in\left(\mathbb{R}_{+}^{L}\right)^{\mathbb{D}} \times\left(\mathbb{R}_{+}^{\mathbb{D}^{-}}\right)^{J}$, which satisfies Conditions (7), (8) and (9) of Lemma 1 with respect to $p \in H^{\mathbb{D}}, q \in \mathbb{R}^{\mathbb{D}^{-}}$and $y \in \prod_{j \in \mathcal{J}} Y^{j}$. Then, $\left(\bar{x}^{i}, \bar{a}^{i}\right)$ belongs to the budget set $B^{i}(p, q, y)$. We first remark that for all $x^{i} \in P^{i}\left(\bar{x}^{i}\right)$, Condition (8), $P^{i}\left(\bar{x}^{i}\right)$ open and convex and $\left(\pi^{i}(\xi) p(\xi)\right)_{\xi} \cdot \bar{x}^{i}>0$ imply that

$$
\left(\pi^{i}(\xi) p(\xi)\right)_{\xi} \cdot x^{i}>\left(\pi^{i}(\xi) p(\xi)\right)_{\xi} \cdot \bar{x}^{i}>0
$$

From Assumption $\mathbf{C}$, for all $\xi \in \mathbb{D}, \bar{x}^{i}+t \mathbf{1}_{\xi} \in P^{i}\left(\bar{x}^{i}\right)$ for some $t>0$. One then deduces from the previous inequality that $\pi^{i}(\xi)(p(\xi) \bullet \mathbf{\ell})>0$, which implies $\pi^{i}(\xi)>0$. Now, we prove that it is optimal by contradiction. If $\left(\bar{x}^{i}, \bar{a}^{i}\right)$ is not a maximal element of $P^{i}\left(\bar{x}^{i}\right)$ in the budget set $B^{i}(p, q, y)$, there exists $\left(x^{i}, a^{i}\right) \in\left(\tilde{P}^{i}\left(\bar{x}^{i}\right) \cap B^{i}(p, q, y)\right)$ with $\tilde{P}^{i}\left(\bar{x}^{i}\right)=P^{i}\left(\bar{x}^{i}\right) \times\left(\mathbb{R}_{+}^{\mathbb{D}^{-}}\right)^{J}$. Since $\left(x^{i}, a^{i}\right)$ belongs to the budget set, one has

$$
p(\xi) \underset{\ell}{\bullet}\left(x^{i}(\xi)-e^{i}(\xi)-a^{i}\left(\xi^{-}\right) \underset{j}{\bullet} y(\xi)\right)-q(\xi) \underset{j}{\bullet}\left(a^{i}\left(\xi^{-}\right)-a^{i}(\xi)\right) \leq 0, \forall \xi \in \mathbb{D} .
$$

Since $\pi^{i}(\xi) \geq 0$ for all $i$, one gets,

$$
\begin{aligned}
\sum_{\xi \in \mathbb{D}} \pi^{i}(\xi)\left(p(\xi) \underset{\ell}{\bullet}\left(x^{i}(\xi)-e^{i}(\xi)\right)\right) & -\sum_{t=0}^{T} \sum_{\xi \in \mathbb{D}_{t}} \pi^{i}(\xi)\left(p(\xi) \bullet_{\ell}\left(a^{i}\left(\xi^{-}\right) \underset{j}{\bullet} y(\xi)\right)\right. \\
& \left.+q(\xi) \underset{j}{\bullet}\left(a^{i}\left(\xi^{-}\right)-a^{i}(\xi)\right)\right) \leq 0
\end{aligned}
$$

and

$$
\begin{aligned}
\sum_{\xi \in \mathbb{D}} \pi^{i}(\xi)\left(p(\xi) \bullet_{\ell}\left(\bar{x}^{i}(\xi)-e^{i}(\xi)\right)\right) & -\sum_{t=0}^{T} \sum_{\xi \in \mathbb{D}_{t}} \pi^{i}(\xi)\left(p(\xi) \bullet_{\ell}\left(\bar{a}^{i}\left(\xi^{-}\right) \underset{j}{\bullet} y(\xi)\right)\right. \\
& \left.+q(\xi) \underset{j}{\bullet}\left(\bar{a}^{i}\left(\xi^{-}\right)-\bar{a}^{i}(\xi)\right)\right)=0 .
\end{aligned}
$$

Combining the above equality and conditions (9b), one gets

$$
\sum_{\xi \in \mathbb{D}} \pi^{i}(\xi)\left(p(\xi) \mathfrak{\ell}_{\ell}\left(\bar{x}^{i}(\xi)-e^{i}(\xi)\right)\right)-\pi^{i}\left(\xi_{0}\right)\left(a^{i}\left(\xi_{0}^{-}\right) \bullet_{j}\left(\left(p\left(\xi_{0}\right) \bullet_{\ell} y^{j}\left(\xi_{0}\right)\right)_{j \in \mathcal{J}}+q\left(\xi_{0}\right)\right)\right)=0 .
$$

Thus,

$$
\begin{aligned}
\sum_{\xi \in \mathbb{D}} \pi^{i}(\xi)\left(p(\xi) \bullet \ell\left(x^{i}(\xi)-\bar{x}^{i}(\xi)\right)\right) & +\sum_{\xi \in \mathbb{D}^{-}} a^{i}(\xi) \bullet j\left(\left(\pi^{i}(\xi) q^{j}(\xi)\right)_{j \in \mathcal{J}}\right. \\
& \left.-\sum_{\xi^{\prime} \in \xi^{+}} \pi^{i}\left(\xi^{\prime}\right)\left(\left(q^{j}\left(\xi^{\prime}\right)\right)_{j \in \mathcal{J}}+\left(p\left(\xi^{\prime}\right) \bullet \bullet_{\ell} y^{j}\left(\xi^{\prime}\right)\right)_{j \in \mathcal{J}}\right)\right) \leq 0
\end{aligned}
$$


It follows from Condition (9a) and the non-negativity of the shares $a^{i}(\xi)$ in each state that,

$$
a^{i}(\xi) \underset{j}{\bullet}\left(\left(\pi^{i}(\xi) q^{j}(\xi)\right)_{j \in \mathcal{J}}-\sum_{\xi^{\prime} \in \xi^{+}} \pi^{i}\left(\xi^{\prime}\right)\left(q\left(\xi^{\prime}\right)+\left(p\left(\xi^{\prime}\right)_{\ell} y^{j}\left(\xi^{\prime}\right)\right)_{j \in \mathcal{J}}\right)\right) \geq 0 .
$$

From the two previous inequalities, one deduces that,

$$
\left(\pi^{i}(\xi) p(\xi)\right)_{\xi} \cdot x^{i} \leq\left(\pi^{i}(\xi) p(\xi)\right)_{\xi} \cdot \bar{x}^{i} .
$$

Thus, one gets a contradiction with Inequality (12).

\subsection{Proof of Theorem 3.1}

We end this section with the proof of Theorem 3.1. We recall the result of Jofré-Rivera-Cayupi (1998), which is an extension of the result of Cornet-Rockafellar. In the following result, $\partial g$ denotes the generalized gradient of a locally Lipschitz function $g$ (see, Clarke (1983)) and $d_{\mathcal{Z}}$ denotes the distance function to $\mathcal{Z}$.

Theorem 4.1 Let $f: \prod_{k=1}^{n} \mathbb{R}^{\ell_{k}} \longrightarrow \mathbb{R}^{\ell_{0}}$ be a locally Lipschitz mapping, $\left\{\mathcal{Z}^{k}\right\}_{k=1}^{n}$ be a finite family of nonempty closed subsets such that $\mathcal{Z}^{k}$ is a subset of $\mathbb{R}^{\ell_{k}}$ for all $k=1, \ldots, n$. Let $\bar{z}:=\left(\bar{z}^{1}, \ldots, \bar{z}^{n}\right) \in$ $\mathcal{Z}^{1} \times \ldots \times \mathcal{Z}^{n}$ such that $f(\bar{z}) \in \operatorname{Fr}\left[f\left(\mathcal{Z}^{1}, \ldots, \mathcal{Z}^{n}\right)\right]$. Then, there exists $\pi \in \mathbb{R}^{\ell_{0}}, \pi \neq 0$ such that,

$$
0 \in \partial\left(\pi^{t} f\right)(\bar{z})+\prod_{k=1}^{n} \partial d_{\mathcal{Z}^{k}}\left(\bar{z}^{k}\right)
$$

There is a slight difference with the original result since we allow the sets $\mathcal{Z}^{k}$ to be subsets of different Euclidean spaces. The proof is the same with only some changes in the notations. We will actually use in the application of this result the inclusion $\partial d_{\mathcal{Z}^{k}}\left(\bar{z}^{k}\right) \subset N\left(\mathcal{Z}^{k}, \bar{z}^{k}\right)$. We now come to the proof of Theorem 3.1, which is organized as follows: we take as fixed a constrained Pareto optimal allocation $(\bar{x}, \bar{a}, \bar{y})$; in the first step, we define the function $f$, the finite family of nonempty closed sets $\left\{\mathcal{Z}^{k}\right\}_{k=1}^{5}$ and $\bar{z}$, and, we prove that $f(\bar{z})$ belongs to $\operatorname{Fr}(f(\bar{z}))$ using Assumption $\mathbf{C}$; in the second step, we apply Theorem 4.1, and, we compute $\partial\left(\pi^{t} f\right)(\bar{z})$; in the third step, we conclude by showing that we recover the conditions $(i)$ to $(v)$ of Theorem 3.1.

First step. We consider the mapping $f$ from $\left(\mathbb{R}^{L}\right)^{\mathbb{D}^{+}} \times\left(\mathbb{R}^{\mathbb{D}_{0}^{-}}\right)^{J} \times\left(\mathbb{R}^{L \mathbb{D}}\right)^{I} \times\left(\mathbb{R}^{L \mathbb{D}}\right)^{J} \times\left(\mathbb{R}^{\mathbb{D}^{-}}\right)^{I J}$ to $\left(\mathbb{R}^{J}\right)^{\mathbb{D}^{-}} \times \mathbb{R}^{L} \times\left(\mathbf{1}^{\perp}\right)^{\mathbb{D}^{+}} \times\left(\mathbb{R}^{I}\right)^{\mathbb{D}^{+}}$, which associates to

$$
\begin{aligned}
z=\left((p(\xi))_{\xi \in \mathbb{D}^{+}},\left(q^{j}(\xi)\right)_{j \in \mathcal{J}, \xi \in \mathbb{D}_{0}^{-}},\left(x^{i}(\xi)\right)_{i \in \mathcal{I}, \xi \in \mathbb{D}},\left(y^{j}(\xi)\right)_{j \in \mathcal{J}, \xi \in \mathbb{D}},\left(a_{j}^{i}(\xi)\right)_{i \in \mathcal{I}, j \in \mathcal{J}, \xi \in \mathbb{D}^{-}}\right) \\
f(z)=\left(\left(f_{\xi}^{1}(z)\right)_{\xi \in \mathbb{D}^{-}},\left(f_{\xi}^{2}(z)\right)_{\xi \in \mathbb{D}},\left(f_{\xi}^{3, i}(z)\right)_{i \in \mathcal{I}, \xi \in \mathbb{D}^{+}}\right) \text {defined by } \\
f_{\xi}^{1}(z)=\sum_{i \in \mathcal{I}} a^{i}(\xi), \forall \xi \in \mathbb{D}^{-} ; \\
f_{\xi_{0}}^{2}(z)=\sum_{i \in \mathcal{I}} x^{i}\left(\xi_{0}\right)-\sum_{j \in \mathcal{J}} y^{j}\left(\xi_{0}\right) ; \\
f_{\xi}^{2}(z)=\operatorname{Proj}_{1^{\perp}}^{\perp}\left(\sum_{i \in \mathcal{I}} x^{i}(\xi)-\sum_{j \in \mathcal{J}} y^{j}(\xi)\right), \forall \xi \in \mathbb{D}^{+} ;
\end{aligned}
$$

for all $\mathrm{i} \in \mathcal{I}$, 


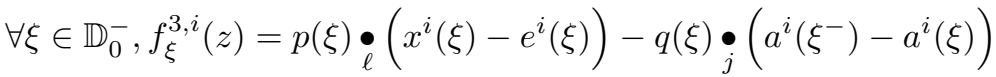

$$
\begin{aligned}
& -p(\xi) \underset{\ell}{\bullet}\left(a^{i}(\xi) \cdot y(\xi)\right) ; \\
& \forall \xi \in \mathbb{D}_{T}, f_{\xi}^{3, i}(z)=p(\xi) \boldsymbol{\bullet}_{\ell}\left(x^{i}(\xi)-e^{i}(\xi)\right)-p(\xi) \underset{\ell}{\bullet}\left(a^{i}(\xi) \underset{j}{\bullet} y(\xi)\right) .
\end{aligned}
$$

From Assumption $\mathbf{C}$, for each $i \in \mathcal{I}$, there exists $\varepsilon^{i}>0$ such that for all $\left.t \in\right] 0, \varepsilon^{i}$, for all $x^{i} \in$ $\bar{B}\left(\bar{x}^{i}, \varepsilon\right) \cap \operatorname{adh} P^{i}\left(\bar{x}^{i}\right), x^{i}+t \mathbf{1}_{\xi_{0}}$ belongs to the interior of $P^{i}\left(\bar{x}^{i}\right)$. Let $\bar{\varepsilon}=\min \left\{\varepsilon^{i} \mid i \in \mathcal{I}\right\}$. Let $\mathcal{Z}^{1}=H^{\mathbb{D}^{+}}, \mathcal{Z}^{2}=\left(\mathbb{R}^{\mathbb{D}_{0}^{-}}\right)^{J}, \mathcal{Z}^{3}=\prod_{i \in \mathcal{I}} \operatorname{adh} P^{i}\left(\bar{x}^{i}\right) \cap \bar{B}\left(\bar{x}^{i}, \bar{\varepsilon}\right), \mathcal{Z}^{4}=\prod_{j \in \mathcal{J}} Y^{j}$ and $\mathcal{Z}^{5}=\left(\mathbb{R}_{+}^{\mathbb{D}^{-}}\right)^{I J}$. Note that $\mathcal{Z}^{3}, \mathcal{Z}^{4}$ are closed subsets and $\mathcal{Z}^{1}, \mathcal{Z}^{2}$ and $\mathcal{Z}^{5}$ are closed convex subsets. We define the vector $w$ as follows :

$$
w:=\left(1_{J^{\mathbb{D}^{-}}}, \sum_{i \in \mathcal{I}} e^{i}\left(\xi_{0}\right),\left(\operatorname{Proj}_{\mathbf{1}^{\perp}}^{\perp}\left(\sum_{i \in \mathcal{I}} e^{i}(\xi)\right)\right)_{\xi \in \mathbb{D}=}, 0_{\mathbb{R}^{I \mathbb{D}}}\right) \in\left(\mathbb{R}^{J}\right)^{\mathbb{D}^{-}} \times \mathbb{R}^{L} \times\left(\mathbf{1}^{\perp}\right)^{\mathbb{D}^{+}} \times\left(\mathbb{R}^{I}\right)^{\mathbb{D}^{+}} .
$$

Let $(\bar{x}, \bar{a}, \bar{y})$ be the constrained Pareto optimum that we consider and let $\bar{p}$ and $\bar{q}$ be the spot prices and the stock prices, which finance it. One easily checks that $\bar{z}=(\bar{p}, \bar{q}, \bar{x}, \bar{y}, \bar{a})$ satisfies $w=f(\bar{z})$ and $\bar{z} \in \prod_{k=1}^{5} \mathcal{Z}^{k}$. We now prove that $w \in \operatorname{Frf}\left(\prod_{k=1}^{5} \mathcal{Z}^{k}\right)$. For all $i \in \mathcal{I}$, let

$$
\mathrm{v}^{i}=\left(0_{J^{\mathbb{D}^{-}}}, \mathbf{1},(0)_{\xi \in \mathbb{D}^{+}}, 0_{\mathbb{R}^{I \mathbb{D}}}\right) \in\left(\mathbb{R}^{J}\right)^{\mathbb{D}^{-}} \times \mathbb{R}^{L} \times\left(\mathbf{1}^{\perp}\right)^{\mathbb{D}^{+}} \times\left(\mathbb{R}^{I}\right)^{\mathbb{D}^{+}} .
$$

To prove the assertion, we show that $\left[w-t \sum_{i \in \mathcal{I}} \mathrm{v}^{i}\right] \notin f\left(\prod_{k=1}^{5} \mathcal{Z}^{k}\right)$ for all $\left.t \in\right] 0, \bar{\epsilon}[$. Let us assume by contraposition that there exists $t \in] 0, \bar{\epsilon}\left[\right.$ such that $\left[w-t \sum_{i \in \mathcal{I}} \mathrm{v}^{i}\right] \in f\left(\prod_{k=1}^{5} \mathcal{Z}^{k}\right)$. Then it exists $z=(p, q, x, y, a) \in \prod_{k=1}^{5} \mathcal{Z}^{k}$ such that $\left[w-t \sum_{i \in \mathcal{I}^{\mathrm{v}}} \mathrm{v}^{i}\right] \in f(z)$.

From the definition of $f$, this implies:

$$
\begin{aligned}
& \sum_{i \in \mathcal{I}} a_{j}^{i}(\xi)=1, \forall \xi \in \mathbb{D}^{-}, \forall j \in \mathcal{J} ; \\
& \sum_{i \in \mathcal{I}}\left(e^{i}\left(\xi_{0}\right)-t \mathbf{1}\right)=\sum_{i \in \mathcal{I}} x^{i}\left(\xi_{0}\right)-\sum_{j \in \mathcal{J}} y^{j}\left(\xi_{0}\right) ; \\
& \operatorname{Proj}_{1^{\perp}}^{\perp}\left(\sum_{i \in \mathcal{I}} e^{i}(\xi)\right)=\operatorname{Proj}_{1^{\perp}}^{\perp}\left(\sum_{i \in \mathcal{I}} x^{i}(\xi)-\sum_{j \in \mathcal{J}} y^{j}(\xi)\right), \forall \xi \in \mathbb{D}^{+} ; \\
& \forall i \in \mathcal{I} \text {, }
\end{aligned}
$$

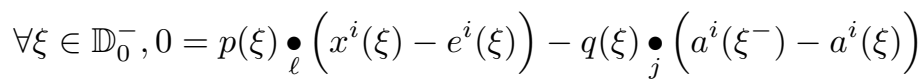

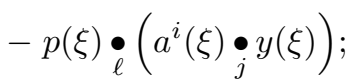

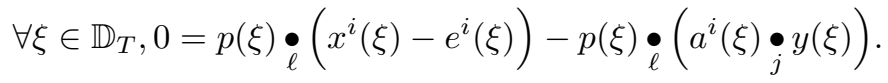

For all $i \in \mathcal{I}$, let $x^{i \prime}=x^{i}+t \mathbf{1}_{\xi_{0}}$. Then, Assumption $\mathbf{C}$ and the choice of $\bar{\epsilon}$ imply that $x^{i \prime} \in P^{i}\left(\bar{x}^{i}\right)$, for all $i \in \mathcal{I}$. Furthermore, the above conditions implies that $\left(x^{\prime}, a, y\right)$ is constrained feasible. Indeed, it suffices to check that $0=\sum_{i \in \mathcal{I}}\left(x^{i \prime}(\xi)-e^{i}(\xi)\right)-\sum_{j \in J} y^{j}(\xi)$ for all $\xi \in \mathbb{D}^{+}$. From the above equalities, we know that the projection on $\mathbf{1}^{\perp}$ is equal to 0 . Then, the vector is proportional to $\mathbf{1}$. From the last equalities, summing over $i \in \mathcal{I}$ and recalling that $\sum_{i \in \mathcal{I}} a_{j}^{i}(\xi)=1$, one gets, $p(\xi) \bullet \sum_{i \in \mathcal{I}}\left(x^{i \prime}(\xi)-\right.$ 
$\left.\left.e^{i}(\xi)\right)-\sum_{j \in J} y^{j}(\xi)\right)=0$. Since $p(\xi) \bullet \ell \mathbf{1}=1$, one deduces that $0=\sum_{i \in \mathcal{I}}\left(x^{i \prime}(\xi)-e^{i}(\xi)\right)-\sum_{j \in J} y^{j}(\xi)$. Finally, one gets a contradiction with the fact that $(\bar{x}, \bar{a}, \bar{y})$ is a constrained Pareto optimum.

Second step. From the previous step and Theorem 4.1, one deduces that there exists $\pi=$ $\left(\left(v^{j}(\xi)\right)_{j \in \mathcal{J}}, p\left(\xi_{0}\right),(\sigma(\xi))_{\xi \in \mathbb{D}^{+}},\left(\pi^{i}(\xi)\right)_{i \in \mathcal{I}, \xi \in \mathbb{D}^{+}}\right), \pi \neq 0$, such that $\xi \in \mathbb{D}^{-}$

$$
\begin{aligned}
0 \in \partial\left(\pi^{t} f\right)(\bar{z}) & +N\left(H^{\mathbb{D}^{+}}, \bar{p}\right) \times \prod_{j \in \mathcal{J}} N\left(\mathbb{R}^{\mathbb{D}_{0}^{-}}, \bar{q}^{j}\right) \times \prod_{i \in \mathcal{I}} N\left(\operatorname{adh} P^{i}\left(\bar{x}^{i}\right) \cap \bar{B}\left(\bar{x}^{i}, \bar{\epsilon}\right), \bar{x}^{i}\right) \\
& \times \prod_{j \in \mathcal{J}} N\left(Y^{j}, \bar{y}^{j}\right) \times \prod_{j \in \mathcal{J}} N\left(\left(\mathbb{R}_{+}^{\mathbb{D}^{-}}\right)^{I}, \bar{a}_{j}\right) .
\end{aligned}
$$

Since $f$ is differentiable, $\partial\left(\pi^{t} f\right)(\bar{z})={ }^{t} D f(\bar{z})(\pi)$. Since $\bar{x}^{i}$ belongs to the interior of $\bar{B}\left(\bar{x}^{i}, \bar{\varepsilon}\right)$,

$$
N\left(\operatorname{adh} P^{i}\left(\bar{x}^{i}\right) \cap \bar{B}\left(\bar{x}^{i}, \bar{\epsilon}\right), \bar{x}^{i}\right)=N\left(\operatorname{adh} P^{i}\left(\bar{x}^{i}\right), \bar{x}^{i}\right) .
$$

Now, we compute some normal cones that are, $N\left(H^{\mathbb{D}^{+}, \bar{p}}\right)=(\mathbb{R} \mathbf{1})^{\mathbb{D}^{+}}, N\left(\mathbb{R}^{\mathbb{D}_{0}^{-}}, \bar{q}^{j}\right)=\left\{\left\{0_{\mathbb{D}_{0}^{-}}\right\}\right\}$and

$$
N\left(\left(\mathbb{R}_{+}^{\mathbb{D}^{-}}\right)^{I}, \bar{a}_{j}\right)=\left\{\left(\nu_{j}^{i}(\xi)\right)_{\substack{i \in \mathcal{I} \\ \xi \in \mathbb{D}^{-}}} \in-\left(\mathbb{R}_{+}^{\mathbb{D}^{-}}\right)^{I} \mid \nu_{j}^{i}(\xi) \bar{a}_{j}^{i}(\xi)=0, \forall \xi \in \mathbb{D}^{-}\right\} .
$$

To compute ${ }^{t} D f(\bar{z})(\pi)$, we use the following property: for all $\gamma \in \prod_{k=1}^{5} \mathbb{R}^{\ell_{k}}$, for all $\pi \in \mathbb{R}^{\ell_{0}}$,

$$
\pi \cdot D f(\bar{z})(\gamma)=\gamma \cdot{ }^{t} D f(\bar{z})(\pi) .
$$

Let us compute $D F(\bar{z})(\gamma)$ for $\gamma=(\beta, \kappa, \alpha, \zeta, \theta) \in H^{\mathbb{D}} \times\left(\mathbb{R}^{\mathbb{D}_{0}^{-}}\right)^{J} \times\left(\mathbb{R}^{L \mathbb{D}}\right)^{I} \times\left(\mathbb{R}^{L \mathbb{D}}\right)^{J} \times\left(\mathbb{R}^{\mathbb{D}^{-}}\right)^{I J}$ :

$$
D f(\bar{z})(\gamma)=\left(\left(D f_{\xi}^{1}(\bar{z})(\gamma)\right)_{\xi \in \mathbb{D}^{-}},\left(D f_{\xi}^{2}(\bar{z})(\gamma)\right)_{\xi \in \mathbb{D}},\left(D f_{\xi}^{3, i}(\bar{z})(\gamma)\right)_{i \in \mathcal{I}, \xi \in \mathbb{D}^{+}}\right),
$$

with

$$
\begin{aligned}
& D f_{\xi}^{1}(\bar{z})(\gamma)=\sum_{i \in \mathcal{I}} \theta^{i}(\xi), \forall \xi \in \mathbb{D}^{-} \\
& D f_{\xi_{0}}^{2}(\bar{z})(\gamma)=\sum_{i \in \mathcal{I}} \alpha^{i}\left(\xi_{0}\right)-\sum_{j \in \mathcal{J}} \zeta^{j}\left(\xi_{0}\right) \\
& D f_{\xi}^{2}(\bar{z})(\gamma)=\operatorname{Proj}_{1^{\perp}}^{\perp}\left(\sum_{i \in \mathcal{I}} \alpha^{i}\left(\xi_{0}\right)-\sum_{j \in \mathcal{J}} \zeta^{j}\left(\xi_{0}\right)\right), \forall \xi \in \mathbb{D}^{+} ;
\end{aligned}
$$

and for all $i \in \mathcal{I}, D f_{\xi}^{3, i}(\bar{z})(\gamma)$ is equal to

$$
\left\{\begin{array}{l}
\beta(\xi) \bullet \bullet_{\ell}\left(\bar{x}^{i}(\xi)-e^{i}(\xi)\right)+\bar{p}(\xi) \bullet \alpha^{i}(\xi)-\kappa(\xi) \bullet_{j}\left(\bar{a}^{i}\left(\xi^{-}\right)-\bar{a}^{i}(\xi)\right)-\bar{q}(\xi) \bullet_{j}\left(\theta^{i}\left(\xi^{-}\right)-\theta^{i}(\xi)\right) \\
-\sum_{j \in \mathcal{J}}\left[\beta(\xi) \bullet \ell \bar{a}_{j}^{i}\left(\xi^{-}\right) \bar{y}^{j}(\xi)+\bar{p}(\xi) \bullet \bullet_{\ell} \bar{a}_{j}^{i}\left(\xi^{-}\right) \zeta^{j}(\xi)+\bar{p}(\xi) \bullet \ell \theta_{j}^{i}\left(\xi^{-}\right) \bar{y}^{j}(\xi)\right], \forall \xi \in \mathbb{D}_{0}^{-} \\
\beta(\xi) \bullet_{\ell}\left(\bar{x}^{i}(\xi)-e^{i}(\xi)\right)+\bar{p}(\xi) \bullet \ell \alpha^{i}(\xi)-\sum_{j \in \mathcal{J}}\left[\beta(\xi) \bullet \ell \bar{a}_{j}^{i}\left(\xi^{-}\right) \bar{y}^{j}(\xi)+\bar{p}(\xi) \bullet \bullet_{\ell} \bar{a}_{j}^{i}\left(\xi^{-}\right) \zeta^{j}(\xi)\right. \\
\left.+\bar{p}(\xi) \bullet_{\ell} \theta_{j}^{i}\left(\xi^{-}\right) \bar{y}^{j}(\xi)\right], \forall \xi \in \mathbb{D}_{T} .
\end{array}\right.
$$

Then, $\pi \cdot D f(\bar{z})(\gamma)$ is equal to

$$
\begin{aligned}
\sum_{\xi \in \mathbb{D}^{-}} v(\xi) \bullet_{j} D f_{\xi}^{1}(\bar{z})(\gamma)+p\left(\xi_{0}\right) \bullet \ell f_{\xi_{0}}^{2}(\bar{z})(\gamma) & +\sum_{\xi \in \mathbb{D}^{+}} \sigma(\xi) \bullet \ell f_{\xi}^{2}(\bar{z})(\gamma) \\
& +\sum_{i \in \mathcal{I}} \sum_{\xi \in \mathbb{D}^{+}} \pi^{i}(\xi) D f_{\xi}^{3, i}(\bar{z})(\gamma)
\end{aligned}
$$


From this formula, one deduces that

$$
{ }^{t} D f(\bar{z})(\pi)=\left(\begin{array}{lllll}
A & B & C & D & E
\end{array}\right)
$$

with

$$
\begin{aligned}
& A=\left(\sum_{i \in \mathcal{I}} \pi^{i}(\xi)\left(\bar{x}^{i}(\xi)-e^{i}(\xi)-\bar{a}^{i}\left(\xi^{-}\right) \dot{y}^{j}(\xi)\right)\right)_{\xi \in \mathbb{D}^{+}} \\
& B=\left(\sum_{i \in \mathcal{I}} \pi^{i}(\xi)\left(\bar{a}^{i}(\xi)-\bar{a}^{i}\left(\xi^{-}\right)\right)\right)_{\xi \in \mathbb{D}_{0}^{-}} \\
& C=\left(p\left(\xi_{0}\right),\left(\sigma(\xi)+\pi^{i}(\xi) \bar{p}(\xi)\right)_{\xi \in \mathbb{D}^{+}}\right)_{i \in \mathcal{I}} \\
& D=-\left(p\left(\xi_{0}\right),\left(\sigma(\xi)+\sum_{i \in \mathcal{I}} \pi^{i}(\xi) \bar{a}_{j}^{i}\left(\xi^{-}\right) \bar{p}(\xi)\right)_{\xi \in \mathbb{D}^{+}}\right)_{j \in \mathcal{J}} \\
& E=\left(v^{j}\left(\xi_{0}\right)-\sum_{\xi^{\prime} \in \xi_{0}^{+}} \pi^{i}\left(\xi^{\prime}\right)\left(\bar{q}^{j}\left(\xi^{\prime}\right)+\bar{p}\left(\xi^{\prime}\right) \bullet \ell \bar{y}^{j}\left(\xi^{\prime}\right)\right),\right. \\
& \left(v^{j}(\xi)+\pi^{i}(\xi) \bar{q}^{j}(\xi)-\sum_{\xi^{\prime} \in \xi^{+}} \pi^{i}\left(\xi^{\prime}\right)\left(\bar{q}^{j}\left(\xi^{\prime}\right)+\bar{p}\left(\xi^{\prime}\right) \bullet_{\ell} \bar{y}^{j}\left(\xi^{\prime}\right)\right)\right)_{\substack{\xi \in \mathbb{D}_{t} \\
0<t<T-1}}, \\
& \left.\left(v^{j}(\xi)+\pi^{i}(\xi) \bar{q}^{j}(\xi)-\sum_{\xi^{\prime} \in \xi^{+}} \pi^{i}\left(\xi^{\prime}\right)\left(\bar{p}\left(\xi^{\prime}\right) \bullet \ell \bar{y}^{j}\left(\xi^{\prime}\right)\right)\right)_{\xi \in \mathbb{D}_{T-1}}\right)_{\substack{i \in \mathcal{I} \\
j \in \mathcal{J}}} .
\end{aligned}
$$

Combining Equations (16) and (19), one gets Conditions $(i)$ to $(v)$ of Theorem 3.1. For Equation (3), for all $\xi \in \mathbb{D}^{+}$, there exists $\lambda(\xi) \in \mathbb{R}$ such that

$$
\sum_{i \in \mathcal{I}} \pi^{i}(\xi)\left(\bar{x}^{i}(\xi)-e^{i}(\xi)-\bar{a}^{i}\left(\xi^{-}\right) \bullet \bar{y}^{j}(\xi)\right)=\lambda(\xi) \mathbf{1}
$$

But, one remarks that for all $i$, for all $\xi \in \mathbb{D}_{0}^{-}$,

$$
\bar{p}(\xi) \underset{\ell}{\bullet}\left(\bar{x}^{i}(\xi)-e^{i}(\xi)-\bar{a}^{i}\left(\xi^{-}\right) \underset{j}{\bullet} \bar{y}^{j}(\xi)\right)=\bar{q}(\xi) \underset{j}{\bullet}\left(\bar{a}^{i}\left(\xi^{-}\right)-a^{i}(\xi)\right),
$$

and the second member of the equality is equal to 0 if $\xi \in \mathbb{D}_{T}$. Consequently,

$$
\bar{p}(\xi) \sum_{\ell} \sum_{i \in \mathcal{I}} \pi^{i}(\xi)\left(\bar{x}^{i}(\xi)-e^{i}(\xi)-\bar{a}^{i}\left(\xi^{-}\right) \underset{j}{\bullet} \bar{y}^{j}(\xi)\right)=\bar{q}(\xi) \bullet_{j} \sum_{i \in \mathcal{I}} \pi^{i}(\xi)\left(\bar{a}^{i}\left(\xi^{-}\right)-a^{i}(\xi)\right)=0 .
$$

The second equality comes from the condition $B=0$. Hence $\bar{p}(\xi) \bullet \ell(\lambda(\xi) \mathbf{1})=\lambda(\xi)=0$, which leads to Equation (3).We end the proof by showing that there exists $i \in \mathcal{I}$ such that $p^{i} \neq 0$. If $p\left(\xi_{0}\right), \pi^{i}(\xi)$ and $\sigma(\xi)$ are all equal to 0 for all $i$ and $\xi$, then, using Equations (6), one gets $v=0$ since for all $j$ and for all $\xi$, there exists at least a consumer $i$ such that $\bar{a}_{j}^{i}(\xi)>0$. This contradicts $\pi \neq 0$. Hence $p\left(\xi_{0}\right)$, $\pi^{i}(\xi)$ and $\sigma(\xi)$ are not all equal to 0 for all $i$ and $\xi$. Consequently, $p^{i}$ is not equal to 0 for at least one consumer. From Assumption $\mathbf{C}, \mathbf{1}_{\xi}$ belongs to $T\left(\operatorname{adh} P^{i}\left(\bar{x}^{i}\right), \bar{x}^{i}\right)$. Since $p^{i} \in-N\left(\operatorname{adh} P^{i}\left(\bar{x}^{i}\right), \bar{x}^{i}\right)$, one gets $p^{i} \cdot \mathbf{1}_{\xi} \geq 0$. Since $\sigma(\xi) \in \mathbf{1}^{\perp}$, one gets $p\left(\xi_{0}\right) \bullet_{\ell} \mathbf{1} \geq 0$ and $\pi^{i}(\xi) \geq 0$ for all $i$ and $\xi$. 


\section{References}

[1] Aliprantis, C.D., B. Cornet and R. Tourky, (2002), "Economic Equilibrium: Optimality and Price Decentralization", Positivity, 6, 205-241.

[2] Bejan C., (2003), "The Objective of a Privately Owned Firm under Imperfect Competition", Working Paper, University of Minnesota.

[3] Bonnisseau, J.M., and O. Lachiri, (2002), Drèze's Criterion in a Multi-Period Economy With Stock Markets, Cahier de la MSE 2002-98, Université Paris 1.

[4] Clarke, F. (1983), Optimization and nonsmooth analysis. Wiley, N.Y.

[5] Diamond, P.A., (1967), "The Role of a Stock Market in a General equilibrium Model with Technological Uncertainty", American Economic Review, 57, 759-776.

[6] Dierker, E., H. Dierker and B. Grodal, (2001), "Objectives of an Imperfectly Competitive Firm: A surplus Approach," in G. Debreu, W. neuefeind, and W. trockel (eds.), Economic Essays, A festschrift for Werner Hildenbrand, Springer, Berlin 2001, 59-81.

[7] Drèze, J.H. (1974), "Investment under Private ownership: Optimality, Equilibrium and Stability", in Allocation Under Uncertainty; Equilibrium and Optimality, J.H. Drèze ed., New-York, Wiley, 129-165.

[8] Geanakoplos, J., J. Drèze, M. Magill and M. Quinzii (1990), "Generic Inefficiency of Stock Market Equilibium When Markets Are Incomplete", Journal of Mathematical, 19, 113-151.

[9] Guesnerie, R. (1975), "Pareto Optimality in Non-convex Economies", Econometrica, 43, $1-31$.

[10] Grossman, S. and O. Hart (1979), "A Theory of Competitive Equilibrium in Stock Market Economies", Econometrica, 47, 293-330.

[11] Jofré, A. and J. Rivera, (1999), "A nonconvex separation property and applications". To appear in Mathematical Programing.

[12] Magill, M. and M. Quinzii (1996), Theory of Incomplete Markets, Cambridge, London: MIT Press. 